\title{
The efficacy of etanercept as anti-breast cancer treatment is attenuated by residing macrophages
}

\author{
Elnaz Shirmohammadi ${ }^{1}$, Seyed-Esmaeil Sadat Ebrahimi ${ }^{1}$, Amir Farshchi $^{2}$ and Mona Salimi ${ }^{3^{*}}$ (D)
}

\begin{abstract}
Background: Interaction between microenvironment and breast cancer cells often is not considered at the early stages of drug development leading to failure of many drugs at later clinical stages. Etanercept is a TNF-alpha inhibitor that has been investigated for potential antitumor effect in breast cancer with conflicting results.

Methods: Secretome data on MDA-MB-231 cancer cell-line were from public repositories and subjected to gene enrichment analyses. Since MDA-MB-231 cells secrete high levels of Granulocyte-Monocyte Colony Stimulating Factor, which activates macrophages to promote tumor growth, the effect of macrophage co-culturing on anticancer efficacy of Etanercept in breast cancer was evaluated using the Boolean network modeling and in vitro experiments including invasion, cell cycle, Annexin PI, and tetrazolium based viability assays and NFKB activity.

Results: The secretome profile of MDA-MB-231 cells was similar to the expression of genes following treatment of breast cancer cells with TNF-a. Accordingly, inhibition of TNF-a by Etanercept decreased MDA-MB-231 cell survival, induced apoptosis and cell cycle arrest in vitro and inhibited NFKB activation. The inhibitory effect of Etanercept on cell viability, cell cycle progression, invasion and induction of apoptosis decreased following co-culturing of the cancer cells with macrophages. The Boolean network modeling of the changes in the dynamics of intracellular signaling pathways revealed NFKB activation by secretome of macrophages, leading to a decreased efficacy of Etanercept, suggesting NFKB inhibition as an alternative approach to inhibit cancer cell growth in the presence of macrophage crosstalk.

Conclusion: This study indicates that the effect of Etanercept may be influenced by residing macrophages in tumor microenvironment, and suggests a method to predict the effect of drugs in the presence of stromal cells to guide experimental designs in drug development.
\end{abstract}

Keywords: TNF- $a$, Etanercept, Breast cancer, Macrophages, Systems biology

\section{Background}

Crosstalk between cancerous and stromal cells in an inflammatory-based microenvironment not only affects the aggressive behavior of cancer cell, but also influences the efficacy of anti-cancer drugs [1-4]. On the other

\footnotetext{
* Correspondence: salimimona@pasteur.ac.ir

${ }^{3}$ Physiology and Pharmacology Department, Pasteur Institute of Iran, P.O. Box: 13164, Tehran, Iran

Full list of author information is available at the end of the article
}

hand, drug impacts are usually assayed in cell culture or immunosuppressed mice models [5], neither of which provides a clear picture of the complicated interactions between tumor cells and their surrounding inflammatory stroma, eventually resulting in inconsistencies in the outcome of clinical trials [6]. It is thus naïve to assess the sole effect of inhibiting a drugable target in the aggressive cancer cells while ignoring the context of micro-environmental changes [4]. Communication 
between cancer cells and their surrounding stroma is through secretion by these cells of soluble factors including growth factors, chemokines and cytokines [7]. The secreted factors reprogram the surrounding stroma with the aim of neutralizing the impact of various intruders disrupting the survival of the cancer cells $[8,9]$.

One of the most influential cytokines in tumor microenvironment is Tumor Necrosis Factor-alpha (TNF- $\alpha$ ) $[10,11]$, long known for its dual effects cancer [12]. Depending on the cellular context as well as its concentration, the effects of TNF- $\alpha$ may vary from inducing necrosis to survival benefits, endowing invasive properties and driving epithelial-mesenchymal transition (EMT) [13].

Considering the pleiotropic and conflicting effects of TNF- $\alpha$, various efforts have been made to exploit this cytokine as a therapeutic target in cancer. In this regard, some approaches have used TNF- $\alpha$ administration to tumor tissue to induce cell death due to its necrotizing effects [14]. However, using TNF- $\alpha$ as a necrotizing agent has been associated with significant lethal side effects limiting the applicability of TNF- $\alpha$ administration to patients [15]. On the other side, TNF- $\alpha$ activates NFKB transcription factor along with JNK and p38 in a cellular context-dependent manner [16]. This canonical pathway induces the expression of several downstream targets of NFKB leading to the increased invasiveness of cancer cells. A different approach that has attracted a great of attention is the use of TNF- $\alpha$ inhibitors, such as Etanercept, as candidates for cancer biotherapy [17].

Etanercept is a dimeric soluble receptor for TNF- $\alpha$, which competes with the cell surface receptors For TNF binding. A phase II clinical trial evaluating the therapeutic efficacy of Etanercept in patients with advanced metastatic breast cancer revealed a reduction in the concentration of TNF- $\alpha$ related cytokines such as CCL2; however, disease stabilization was detected in only one patient [18]. In order to elucidate the underlying reason for this outcome, we aimed at integrating molecularcellular experiments with systems modeling approaches to compare the effect of Etanercept in breast cancer cells alone and in the context of host immune cells.

\section{Methods}

\section{High through-put data analysis and enrichment-based} tests

Proteomics data providing the secretome of MDA-MB231 cells were extracted from GSE51938 dataset in GEO. The secretion profile was submitted to Enrichr online web (http://amp.pharm.mssm.edu/Enrichr/). "LINCS L1000 Ligand Perturbations up" library was used for enrichment analysis. This library consists of gene expression changes induced by various ligands (e.g. growth factors and cytokines) and displays ligands inducing similar gene expression profiles.

Gene-set enrichment tests including gene ontology (GO) biological process, transcription factors and signaling pathways were carried out using the corresponding libraries embedded in Enrichr online web tool.

\section{Boolean network simulation}

Cellular signaling network was constructed based on the literature survey [19] and translated into Boolean rules. Asynchronous simulation mode was used to predict the effect of macrophages on the anti-cancer efficacy of Etanercept. BoolNet R package was used to perform the dynamic modeling and analysis of attractor states [20].

\section{Cell culture and condition medium preparation}

MDA-MB-231 human breast cancer cell line and THP-1 human monocytic cells were purchased from Pasteur Institute of Iran (Tehran, Iran). A total of $2 \times 10^{5}$ THP-1 cells were seeded in $2 \mathrm{ml}$ RPMI supplemented with $10 \%$ FBS in the upper chamber of 6-well insert plates (SPL Life Sciences, Seoul, South Korea). Cells were treated with $10 \mathrm{ng} / \mathrm{ml}$ of PMA (Phorbol 12-myristate 13-acetate) for $24 \mathrm{~h}$ to allow differentiation of THP-1 cells to M0 macrophages and their subsequent attachment to the inserts. After $24 \mathrm{~h}, 2 \times 10^{5}$ of MDA-MB-231 cells were seeded in the lower chamber in $2 \mathrm{ml}$ of DMEM. The coculturing system was kept in 5\% CO2 incubator for $72 \mathrm{~h}$.

To prepare condition medium (CM), the upper insert was withdrawn and MDA-MB-231 cells were washed 3 times with PBS and starved for $24 \mathrm{~h}$ in the serum free DMEM. The condition medium was then filtered using $0.2 \mu \mathrm{m}$ filter and kept at $-70^{\circ} \mathrm{C}$ until use. $\mathrm{CM}$ was diluted with $50 \%$ of fresh medium for further experiments.

\section{Tetrazolium-based viability test}

Co-culturing of MDA-MB-231 with THP-1 cells was performed as described in the previous section. Cocultured as well as MDA-MB-231 cells were treated with 1 or $10 \mu \mathrm{g} / \mathrm{ml}$ of Etanercept (Aryogen Pharmed, Tehran, Iran) or control buffer for $72 \mathrm{~h}$. The concentration of Etanercept was selected based on the plasma concentration obtained after weekly administration of the drug to the arthritis rheumatoid patients [7]. After $72 \mathrm{~h}$, THP-1 containing inserts were discarded, and $20 \mu \mathrm{l}$ of $5 \mathrm{mg} / \mathrm{ml}$ of MTT (3-(4,5-dimethylthiazol-2-yl)-2,5-diphenyltetrazolium bromide) stock solution was added to each well. Following $3 \mathrm{~h}$ of incubation, the supernatants were aspirated, and $200 \mu \mathrm{l}$ of dimethylsulfoxide (DMSO) were used to dissolve purple formazan in each well. Absorbance was measured at $545 \mathrm{~nm}$ using microplate Reader (Stat Fax-2100, ST. Louis, USA). 


\section{Cell cycle analysis}

MDA-MB-231 cells were harvested after $72 \mathrm{~h}$ of coculturing with the differentiated macrophages in the presence and absence of 1 or $10 \mu \mathrm{g} / \mathrm{ml}$ Etanercept. Cells were washed 3 times with cold PBS, detached using $0.025 \%$ trypsin and re-suspended in $70 \%$ ethanol and stored at -20 for at least $24 \mathrm{~h}$ to allow cell fixing. Cells were pelleted by centrifugation at $\times 400 \mathrm{~g}$ and ethanol was discarded. They were then stained with $200 \mu \mathrm{l}$ of PI (Propidium Iodide) $(1 \mathrm{mg} / \mathrm{ml})$ stock solution supplemented with $150 \mu \mathrm{l}$ of stock solution of DNAse-free RNAse A (CinaClone, Tehran. Iran) $(2 \mathrm{mg} / \mathrm{ml})$ and $0.1 \%$ Triton X-100 in $10 \mathrm{ml}$ PBS, and then analyzed by Flow cytometry (PARTEC GmbH, Munster, Germany). FlowJo software was used for data analysis and measuring fraction of the cells in four stages of the cell cycle, i.e. G1, S, G2 and subG1.

\section{Annexin-PI apoptosis assay}

To measure the percentage of apoptotic cells induced by Etanercept either in MDA-MB-231 cells alone or cocultured with the macrophages, Annexin- $V$ staining with PI was used based on the manufacture's instruction. Briefly, cells were washed with PBS and harvested with $0.025 \%$ trypsin. Detached cells were centrifuged at $400 \times g$ for $5 \mathrm{~min}$ and re-suspended in Annexin-PI solution containing $2 \mu \mathrm{l}$ annexin-V-FLUOS labeling agent, $2 \mu \mathrm{l}$ PI solution and $1 \mathrm{ml}$ incubation buffer, and incubated at $37^{\circ} \mathrm{C}$ for $15 \mathrm{~min}$, and then analyzed by flow cytometry.

\section{NFKB activity determination}

A total of $1 \times 10^{6}$ MDA-MB-231 cells were seeded into $100 \mathrm{~mm}$ cell culture plates and allowed to attach for 24 h. Cells were then treated with $50 \%$ diluted CM or normal medium as control for $72 \mathrm{~h}$. Activity of NFKB was determined using Trans $\mathrm{AM}^{\circ}$ NFkB Transcription Factor ELISA Kit (\#40096, Active Motif, Belgium) as described in the kit manual. In brief, following the incubation period, cells were washed twice with PBS supplemented with PhosStop phosphatase inhibitor cocktails (Roche, USA) and $0.05 \%$ NP-40. Ultra-centrifugation was used to extract nucleus from the cytoplasmic fraction. The nucleus pellet was further treated with nucleus lysis buffer and centrifuged at $\times 14,000 \mathrm{~g}$ to extract nuclear proteins. Protein assay was performed using Bradford method [21], and $10 \mu \mathrm{g}$ of nuclear extract was added to each well of DNA-coated wells. To calculate the fraction of DNA-attached NFKB, HRP-conjugated anti-phosphop65 primary antibody was added to each well and the fluorescence intensity was measured at $630 \mathrm{~nm}$ (Stat Fax-2100, ST. Louis, USA).
Quantitative real time PCR for the analysis of macrophage cytokines

A total of $1 \times 10^{6}$ of differentiated THP- 1 cells using 10 $\mathrm{ng} / \mathrm{ml}$ of PMA were treated with $\mathrm{CM}$ for $48 \mathrm{~h}$ and incubated at $37^{\circ} \mathrm{C}$. Cells were washed with PBS and lysed using RNX - Plus solution (CinaClone, Tehran, Iran). Total mRNA was extracted and subjected to cDNA synthesis using Revert Aid first strand cDNA synthesis kit (ThermoFisher Scientific, USA) according to the manufacturer's instructions. PCR was carried out using Taqman mastermix [21]. Forward (F) and reverse (R) primer sequences used were as following:

TNF-A-F: 5' -CCCTGACATCTGGAATCTGGAG-3', TNF-A-R: 5' -TCAAGGAAGTCTGGAAACATCTGG-3', CCL22-F: 5'-TGGGTGAAGATGATTCTCAATAAGC-3', CCL22-R: 5' -CTATAATGGCAGGGAGGTAGGG-3', IL10-F: 5'-CTTGCTGGAGGACTTTAAGGGTTAC-3', IL10-R: 5' -CTTGATGTCTGGGTCTTGGTTCTC-3'.

$\Delta \Delta C T$ was calculated and normalized according to the expression of GAPDH using the following primers, GAPDH-F: 5' -ACATCAAGAAGGTGGTGAAGCAG3', GAPDH-R: 5' - GCGTCAAAGGTGGAGGAGTG-3.

\section{Matrigel invasion assay}

MDA-MB-231 cells were grown in CM for $72 \mathrm{~h}$. Cells were washed 3 times with PBS and starved with serumfree DMEM for 24h. 24-well insert plates (SPL Life Sciences, Seoul, South Korea) with $8 \mu \mathrm{m}$ pore size were coated with $1 \mathrm{mg} / \mathrm{ml}$ of matrigel (BD Biosciences, San Jose, USA). After $4 \mathrm{~h}$ of incubation of the coated plates at $37^{\circ} \mathrm{C}$, when a smooth layer was formed on the insert, a total of $1 \times 10^{5}$ starved cells were added to the upper chamber of each well in serum free medium, and the lower chamber was supplemented with DMEM containing $10 \% \mathrm{FBS}$ as attractant. After $24 \mathrm{~h}$, the inserts were fixed in $4 \%$ formaldehyde and stained using $0.05 \%$ crystal violet. Traversed cells across the membrane were visualized under an inverted microscope.

\section{Statistical analysis}

For the enrichment tests, adjusted $p$-value by Bonferroni method $<0.05$ was considered as significant. For experimental assays, GraphPad Prism was used for data analysis. Data represent mean \pm S.E.M from the indicated replicates for each test. ANOVA was used to determine the significance of difference ( $p$-value $<0.05)$.

\section{Results}

In silico analysis of breast cancer cells secretome

Considering the importance of cytokine profile of invasive breast cancer cells in communicating with stromal cells, the secretome of mesenchymal-like breast cancer cells (MDA-MB-231 cells) were initially extracted from the work of Su et al. [22]. The authors used RayBio ${ }^{\circ}$ 
Cytokine Antibody Array and provided their data freely available in GEO database (GSE51938).

The secreted cytokines with an intensity greater than 0.2 were chosen for enrichment-based tests using the Enrichr online web tool [23]. Using up-regulated signatures induced by ligand perturbations in LINCS L1000 module within Enrichr, the list of secreted cytokines from MDA-MB-231 was found to be statistically mimicked when breast cancer cells were treated with IL1 and TNF- $\alpha$ (Table 1). Moreover, the transcription factor enrichment analysis on ChEA database using Enrichr web tool revealed RELA (a component of NFKB transcription factor) as the top enriched transcription factor associated with the secretome of MDA-MB-231 cells (FDR adjusted $p$-value $<0.05)$. These results suggested NFKB as being highly active in the invasive breast cancer cells resulting in the secretion of a number of cytokines similar to those secreted by cells treated with TNF- $\alpha$. They also provided a rationale for using clinically available TNF- $\alpha$ inhibitors as an approach to alter the secretion profile of invasive breast cancer cells.

\section{Anti-TNF therapy inhibited growth of breast cancer cells}

To further validate the in silico findings, in vitro validation experiments was followed in order to explore the effect of Etanercept, as a TNF- $\alpha$ inhibitor, on suppressing the invasive behavior of breast cancer cells. The viability assay revealed a significant decrease in cell survival following treatment of MDA-MB-231 cells with Etanercept in a concentration-dependent manner ( 1 and $10 \mu \mathrm{g} /$ ml) (Fig. 1a). Cell cycle analysis revealed a significant reduction in the number of cells in G1 and G2 phases, and an increase in the number of cells in subG1 phase at both concentrations of Etanercept, collectively indicating an induction of apoptosis in cancer cells (Fig. 1b). Consistently, the Annexin-PI results displayed an elevated number of both early and late apoptotic cells upon treatment with 1 and $10 \mu \mathrm{g} / \mathrm{ml}$ of Etanercept, further confirming the induction of apoptosis (Fig. 1c). These results suggest thatof TNF- $\alpha$ inhibition is capable of reducing cancer cell survival rate.

The next step consisted of measuring p65 translocation to the nucleus of MDA-MB-231 cells, which revealed a significant reduction in the levels of active
NFKB in the nucleus $(p$-value $<0.05)$ following treatment with Etanercept at 1 and $10 \mu \mathrm{g} / \mathrm{ml}$, suggesting that Etanercept can also diminish the activity of NFKB in MDAMB-231 cells (Fig. 1d).

\section{Cytotoxic effect of Etanercept is reduced in the presence of macrophages}

Contrary to our findings on the inhibitory effects of Etanercept on the growth of breast cancer, there is also evidence demonstrating the inefficiency of Etanercept in suppressing the invasive behavior of tumors [24]. To address this discrepancy, following a closer inspection of MDA-MB-231 secretome within GSE51938 dataset revealed that GM-SCF is highly secreted by these cells [22]. This chemokine is an important activator of macrophages in inflamed tissues, also addressed by $\mathrm{Su}$ et al. [22]. These observations would likely justify the resistance to anti -TNF therapy.

A co-culture system was used to measure several biological parameters. It was shown that co-culturing of MDA-MB-231 cells with THP-1, which were differentiated into M0 macrophages by low concentration of PMA, could significantly increase the viability of MDA-MB-231 cells and decrease the efficacy of Etanercept at 1 and $10 \mu \mathrm{g} / \mathrm{ml}$ in inhibiting survival, proliferation and apoptosis induction (Fig. 2a-c). However, $10 \mu \mathrm{g} / \mathrm{ml}$ of Etanercept was more potent than $1 \mu \mathrm{g} / \mathrm{ml}$ to cause G2/M arrest.

Invasion of cancer cells was increased in the presence of macrophages, and Etancercept was able to diminish this effect at $10 \mu \mathrm{g} / \mathrm{ml}$ (Fig. $2 \mathrm{~d}$-e). Besides, the activity of NFKB in breast cancer cells was significantly augmented, and Etanercept was incapable of lowering this effect to the levels detected in the monocultures of cancer cells (Fig. 2f). These findings suggest that the interaction between cancer cells and macrophages maintains NFKB activity at a high level and inhibition of TNF- $\alpha$ alone would be insufficient to lower its activity.

The reduced efficacy of Etanercept in the presence of macrophages can also be predicted using the signaling network modeling. Hence, a network of TNF- $\alpha$ signaling pathway was constructed in the presence and absence of macrophage secretome using KEGG database and the those of Lu et als [19]. In constructing this signaling network, the crosstalk among core cellular pathways including

Table 1 Enrichment of MDA-MB-231 cell line secretome against "up-regulated gene perturbations induced by ligands in LINCs1000" and CHEA library using Enricher web tool

\begin{tabular}{lll}
\hline Index & Name & Adjusted $\boldsymbol{p}$-value \\
\hline 1 & IL1-BT20 & 0.0002643 \\
3 & TNFA-BT20 & 0.0002643 \\
5 & IL1-MDAMB231 & 0.0002643 \\
7 & TNFA-MDAMB231 & 0.003165 \\
1 & RELA_24523406_ChIP-Seq_FIBROSARCOMA_Human & 0.004114 \\
\hline
\end{tabular}



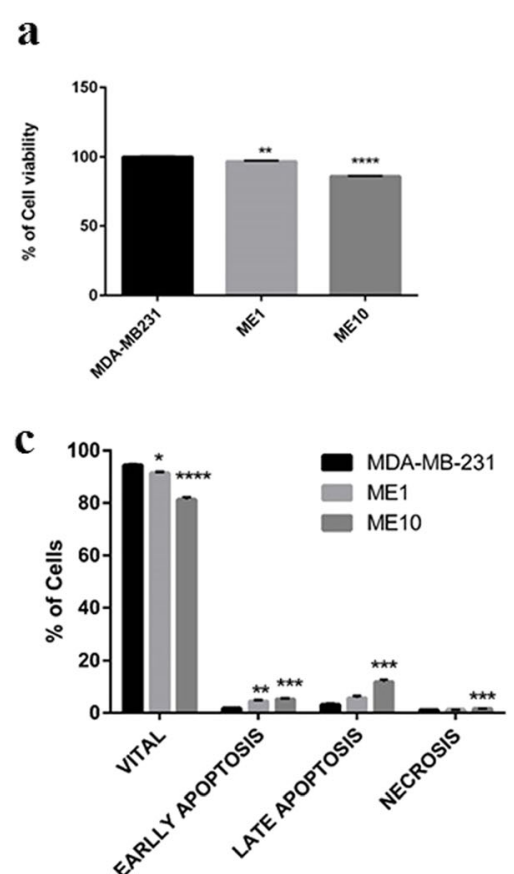

b

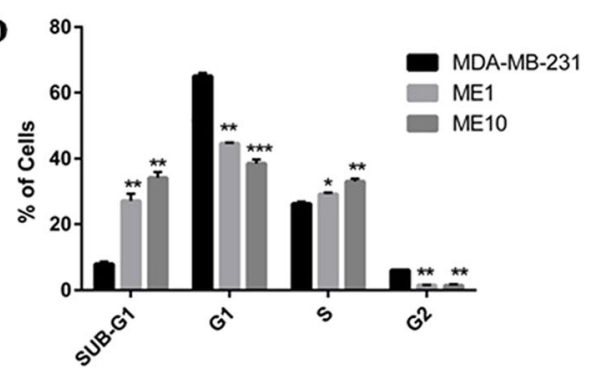

d

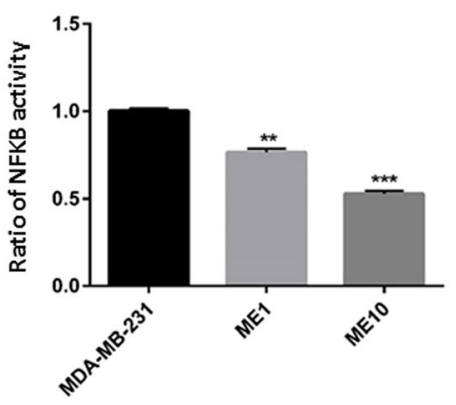

Fig. 1 Assessing the anti-cancer effects of Etanercept as a TNF-a blocker. a MTT viability test in MDA-MB-231 cells following treatment of cells with 1 or $10 \mu \mathrm{g} / \mathrm{ml}$ Etanercept. Data represent mean \pm sem for six replicates. ME1 and ME10 = MDA-MB-231 + 1 or $10 \mu \mathrm{g} / \mathrm{ml}$ Etanercept. b Analysis of cell cycle in MDA-MB-231 cells treated with $1 \mathrm{or} 10 \mathrm{\mu g} / \mathrm{ml}$ Etanercept. Data represent mean \pm sem of number of cells in each phase for 3 replicates. c Measurement of apoptosis using annexin-PI. Data represent mean \pm sem of number of cells in each phase for 3 replicates. $\mathbf{d}$ Measurement of NFKB activity following treatment with Etanercept. Data are normalized to the activity of NFKB in MDA-MB-231 cells as control. ${ }^{*} P<0.05{ }^{* *} P<0.01,{ }^{* * *} P<0.0001$. ME1 and ME10 $=$ MDA-MB-231 +1 or $10 \mu \mathrm{g} / \mathrm{ml}$ Etanercept

NFKB, JAK-STAT and PI3K-AKT was taken into account based on the literature (Fig. 3). The interactions were then translated into a Boolean model to simulate TNF- $\alpha$ signaling in the presence and absence of macrophages. Asynchronous simulation model was used to predict cell fate; i.e. survival, proliferation and apoptosis when TNF- $\alpha$ pathway was active in cancer cells alone versus the condition where macrophages were considered (Supplementary file 1).

Attractor analysis (i.e. the steady state of the system) demonstrated that when breast cancer cells were modeled alone and treated with Etanercept (i.e. Macrophage and TNF- $\alpha$ state were set to 0 ), proliferation and cell survival were found in the OFF state while apoptosis was in the active state. However, in the presence of macrophages (Macrophage $=1$ ) and Etanercept treatment (TNF- $\alpha=0$ ), apoptosis was in the inactive state (apoptosis $=0$ ) and proliferation and survival were still active. These results suggest that Etanercept treatment alone is not effective when macrophage secretome activates additional signaling pathways in the tumors (Fig. 4).

\section{Effect of Etanercept on secretion of TNF-a, IL10 and CCL22 in macrophages}

In order to explore the changes in the function of macrophages following treatment with $\mathrm{CM}$ or Etanercept, the effect of Etanercept on the expression levels of TNF$\alpha$ as well as CCL22, a chemokine marker of M2 phenotype of tumor-associated macrophages contributing to aggressive features of tumors, were measured [25]. It was revealed that the expression level of CCL22 in macrophages grown in $\mathrm{CM}$ for $48 \mathrm{~h}$ was increased compared to cells treated with fresh medium alone, while the same condition slightly decreased the expression of TNF- $\alpha$ (Fig. 5a). In this regard, Etanercept at 1 and $10 \mu \mathrm{g} / \mathrm{ml}$ significantly diminished the expression level of both factors (Fig. 5 b, c).

To extend the list of other co-expressed proteins with CCL22 in CM-treated macrophages, gene co-expression network analysis in THP-1 cells was utilized (Fig. 5d) using Immuno-Navigator web tool (Pearson's correlation coefficient $<0.6$ ) [26]. Thirty nine genes were found to be co-expressed with CCL22 (Supplementary file 2). Among the top co-expressed genes were several cytokines including IL10 along with chemokines such as CCL18 and CXCL13 as well as anti-oxidant enzymes such as SOD2, TXN and TXNRD1 (Fig. 5e). From these top correlated genes, we determined the expression of IL10 following co-culturing and treatment with Etanercept by qPCR. Co-culturing of differentiated macrophages with MDA-MB-231 cells did not significantly 


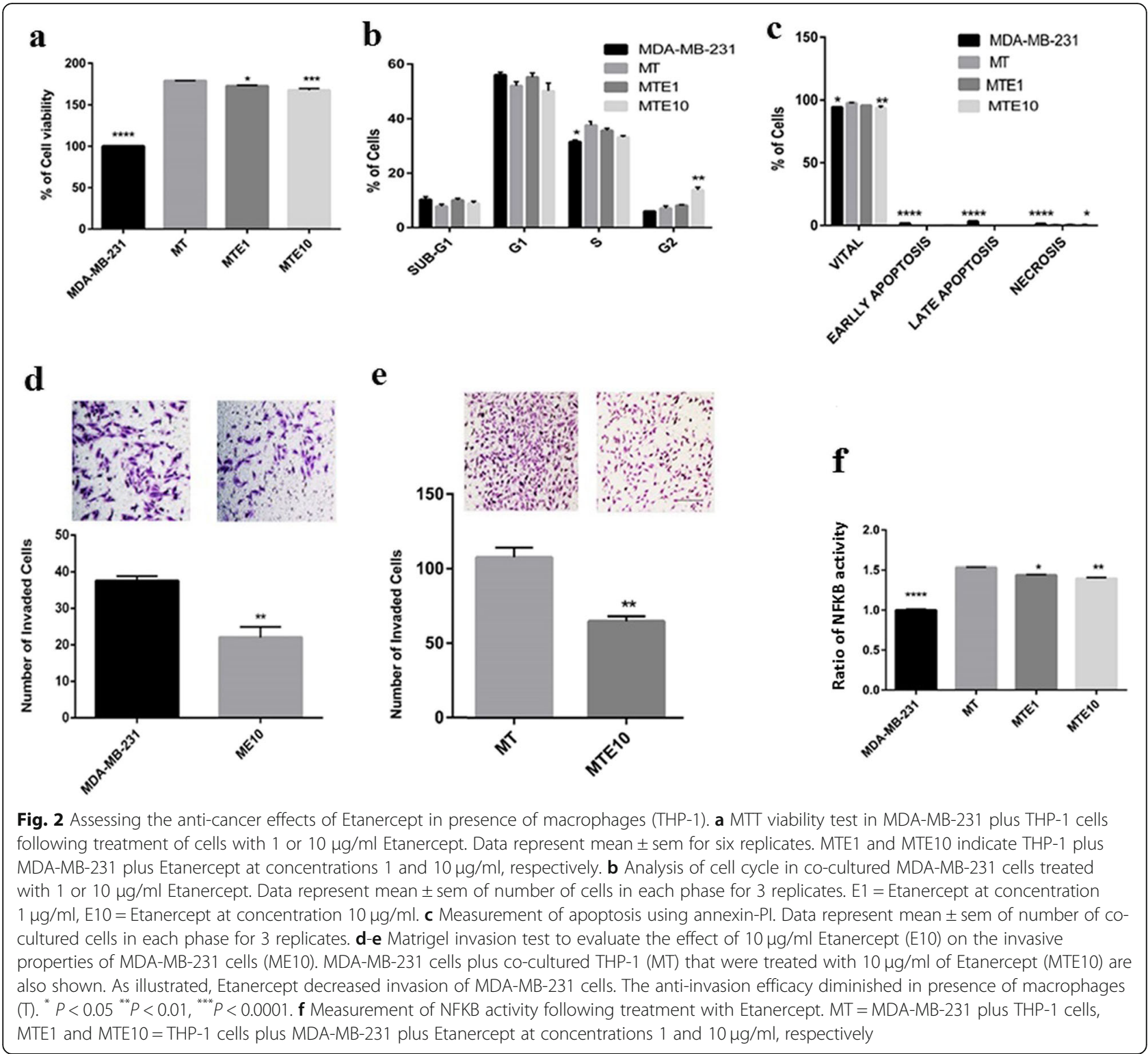

alter the expression level of IL10, likely due to the short co-culturing period used in the study (Fig.5f). However, Etanercept decreased the expression of IL10 at both concentrations of 1 and $10 \mu \mathrm{g} / \mathrm{ml}$ compared to the control vehicle (Fig. 5g,h). These results demonstrated that Etanercept can affect the secretory profile in addition to the function of tumor-associated macrophages; however to a lesser extent.

\section{Discussion}

NFKB is highly active in the invasive breast cancer cells resulting in the secretion of a number of cytokines, a condition similar to that of cells treated with TNF- $\alpha$. These data fueled our initial hypothesis that using clinically available TNF- $\alpha$ inhibitors would be a smart approach to alter the secretion profile of invasive breast cancer cells, and thus motivated us to assess the inhibitory effect of Etanercept, a TNF- $\alpha$ inhibitor, to suppress proliferation and survival of breast cancer cells. Our hypothesis is also supported by the findings of other studies indicating the efficacy of Etanercept, a soluble receptor, to capture and neutralize circulating TNF- $\alpha$ in breast cancer treatment [18, 27]. The present study showed that inhibition of TNF- $\alpha$ signaling by Etanercept results in a reduction in the activity of NFKB transcription factor, leading to reduced survival rates of MDAMB-231 cells, and induced cell cycle arrest and apoptosis.

Notably, the effect of anti-TNF therapies is still controversial with conflicting outcomes at patient level. In this regard, it has been shown that tumor microenvironment exerts profound effects not only on the behavior of 

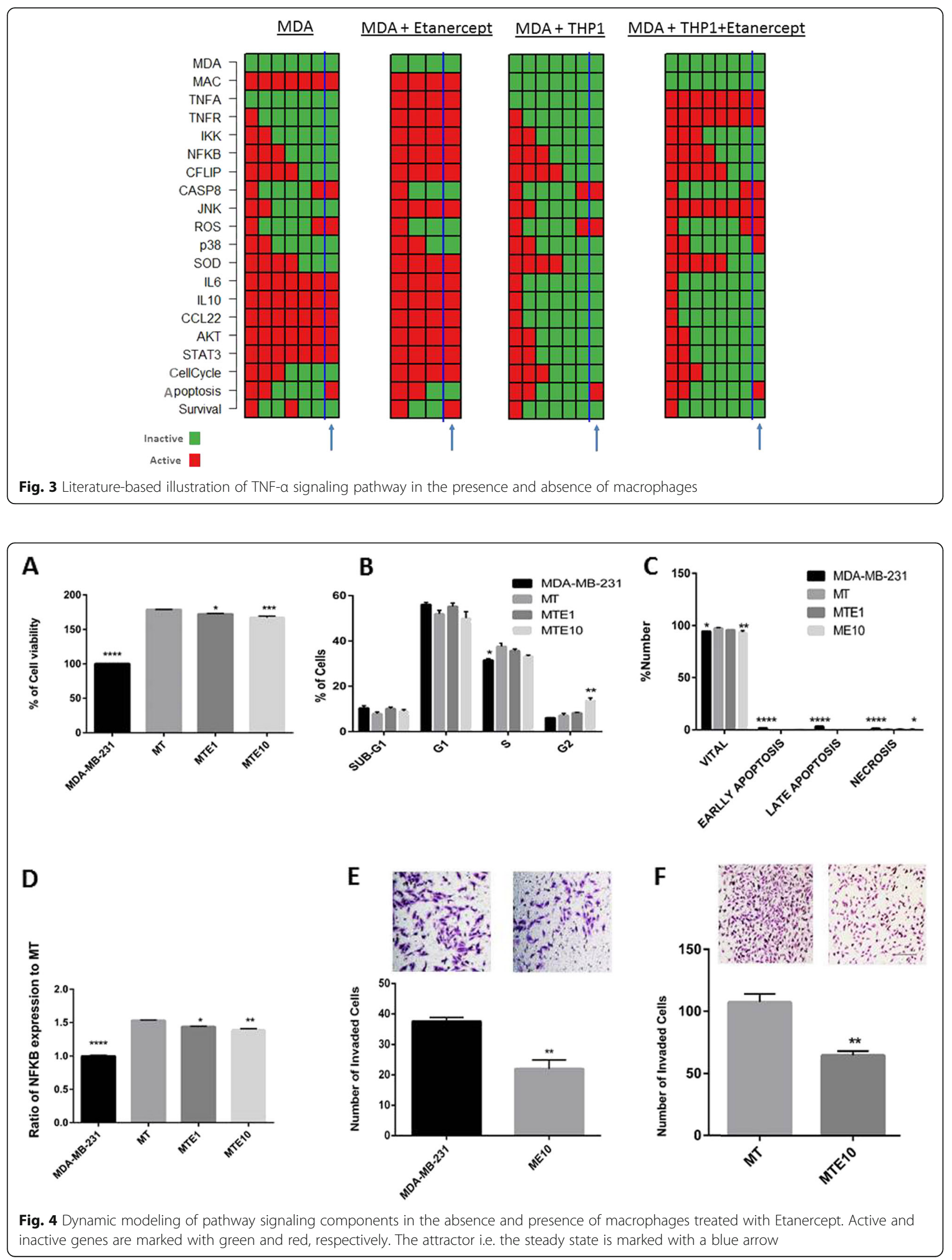


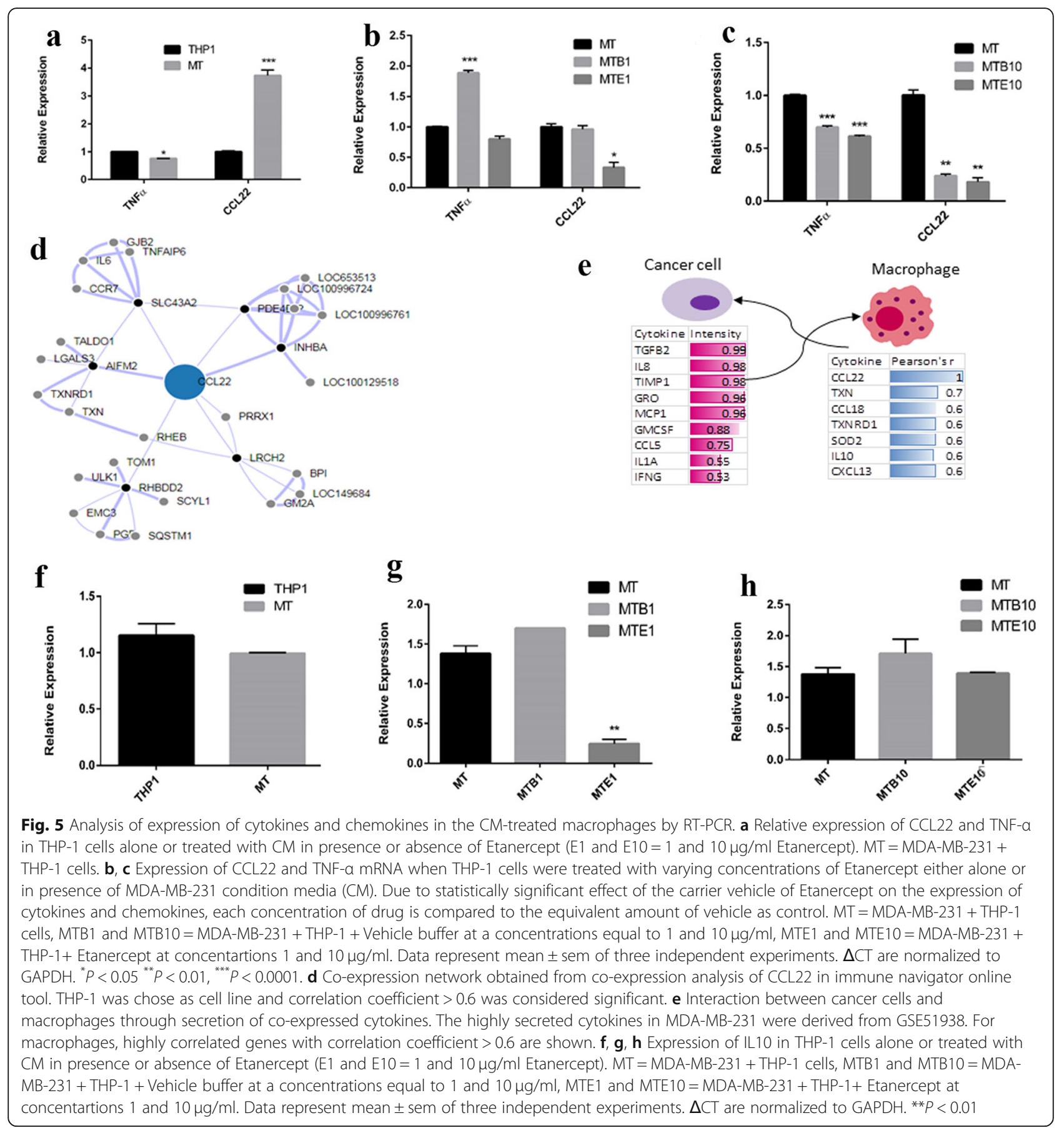

cancer cells but also on the efficacy of treatments that has to be taken into account when evaluating the effect of novel drugs [28]. To address this issue, using publicly available proteomic data of MDA-MB-231 secretome, a high level of Granulocyte-Monocyte Stimulating Factor (GM-CSF) expression was detected in the secretome, which modulate activation of tumor associated macrophages. Accordingly, our findings on cell cycle analysis, annexin/PI and matrigel assays further confirmed that when co-cultured with MDA-MB-231 cells, macrophages reduce the anti-proliferative and antiinvasive effects of Etanercept in cancer cells. These findings suggested that the anti-cancer effect of Etanercept can be diminished or abrogated by resident macrophages in the tumor site, which would in turn suggest a role for the microenvironment in treatment efficiency.

We also observed that in the co-culture condition, Etanercept does not reduce the activity of NFKB to the extent observed in breast cancer cells alone. These results underscore the importance of stromal cell secretome in 
resistance to the targeted therapies. Tumor associated macrophages are important components of tumor stroma, which link inflammation and cancer progression by secreting various cytokines. The secreted cytokines from macrophages can trigger PI3K/AKT/mTOR signaling pathway [29] and also lead to the activation of NFKB as well as STAT3 in cancer cells [30-32]. Interaction between NFKB and STAT3 is a key mediator of crosstalk in the tumor inflammatory microenvironment [33]. STAT3 is constitutively activated in triple negative breast cancer, and ample evidence imply that persistently- activated STAT3 maintains constitutive NFKB activity $[34,35]$. Collectively, these results suggest that the presence of macrophages in tumor tissues potentiates the activity of NFKB resulting in subsequent resistance to anti-TNF therapies. In other words, the activated signaling pathways can decrease the reliance of NFKB activation solely on TNF- $\alpha$. This mechanism may apparently reduce the efficacy of TNF- $\alpha$ inhibition strategy.

Moreover, we used Boolean network modeling to simulate the effect of Etanercept in the presence and absence of macrophages. This modeling provides useful insights to confirm our findings that in the presence of macrophage-associated cytokines and chemokines, activation of JAK-STAT and PI3K-AKT pathways can restore survival and proliferation of tumor cells to compensate the decreased activity of NFKB by Etanercept.

We were also interested to assess the effect of Etanercept on the secretion profile of macrophages that were grown in the CM. We showed that Etanercept reduces the transcript levels of TNF- $\alpha$, IL10 and CCL22, all of which are associated with tumor progression. Thus our results imply that in addition to inhibiting the aggressive behavior of tumor cells within the context of microenvironment, Etanercept is able to alter the activity of tumor associated macrophages.

As a whole, our data suggest that evaluating the anticancer effect of drugs without considering the effect of stromal cells may lead to failure of drug candidates in the later clinical stages. An integrated use of high through-put data available in the literature and systems biology would allow predicting the effect of drugs in the presence of stromal cells. These data should further be validated by in vivo experiments.

\section{Conclusions}

In summary, we concluded that the anticancer efficacy of Etanercept as a TNF-A blocker is influenced by the secretome of the co-cultured macrophages. These results suggest an important effect of stromal cells on the efficacy of targeted drugs in cancer treatment.

\section{Supplementary information}

Supplementary information accompanies this paper at https://doi.org/10. 1186/s12885-020-07228-y.

\section{Additional file 1.}

Additional file 2.

\section{Abbreviations}

GM-CSF: Granulocyte-monocyte colony stimulating factor; LINCS: Library of Integrated Network of Cellular Signaling; TNF1-a: Tumor Necrosis Factor alpha; FADD: Fas-Associated protein with Death Domain; JNK: C-Jun Nterminal Kinase; EMT: Epithelial-Mesenchymal Transition

\section{Acknowledgements}

Authors are grateful to the developers of previous datasets and bioinformatic software which assisted in performing this work.

\section{Authors' contributions}

E.Sh designed and performed the experiments, analyzed data and prepared the manuscript. SE.SE and A. F contributed in preparing the research proposal. M. S was the principle investigator, conceived the idea and helped in the preparation of the manuscript. All authors have read and approved the manuscript.

\section{Funding}

This study was supported by the grant from the Cancer Research Network of Iran to purchase the materials and Biopharmaceutical Research center of ARYOGEN Company to provide us with Etanercept.

\section{Availability of data and materials}

The source code for Boolean network construction and highly correlated genes are provided as supplementary information.

\section{Ethics approval and consent to participate}

All data from extra sources were derived from publicly available data freely provided in the citing manuscripts or public databases that are listed methods and material section.

\section{Consent for publication}

All data from extra sources were derived from publicly available data freely provided in the citing manuscripts or public databases that are listed methods and material section.

\section{Competing interests}

Authors declare no conflicts of interests.

\section{Author details}

${ }^{1}$ School of Pharmacy, International Campus, Tehran University of Medical Sciences, Tehran, Iran. ${ }^{2}$ Biopharmaceutical Research Center, AryoGen Pharmed Inc., Alborz University of Medical Sciences, Karaj, Iran. ${ }^{3}$ Physiology and Pharmacology Department, Pasteur Institute of Iran, P.O. Box: 13164, Tehran, Iran.

Received: 16 July 2019 Accepted: 28 July 2020

Published online: 03 September 2020

References

1. Barneh F, Mirzaie M, Nickchi P, Tan TZ, Thiery JP, Piran M, et al. Integrated use of bioinformatic resources reveals that co-targeting of histone deacetylases, IKBK and SRC inhibits epithelial-mesenchymal transition in cancer. Brief Bioinform. 2019:20(2):717-31.

2. Barneh F, Salimi M, Goshadrou F, Ashtiani M, Mirzaie M, Zali H, et al. Valproic acid inhibits the protective effects of stromal cells against chemotherapy in breast cancer: insights from proteomics and systems biology. J Cell Biochem. 2018:119(11):9270-83.

3. Hazlehurst LA, Landowski TH, Dalton WS. Role of the tumor microenvironment in mediating de novo resistance to drugs and physiological mediators of cell death. Oncogene. 2003;22(47):7396. 
4. Jones VS, Huang R-Y, Chen L-P, Chen Z-S, Fu L, Huang R-P. Cytokines in cancer drug resistance: cues to new therapeutic strategies. Biochimica et Biophysica Acta (BBA)-reviews onCancer. 2016;1865(2):255-65.

5. Calcoen D, Elias L, Yu X. What does it take to produce a breakthrough drug? Nat Rev Drug Discov. 2015;14:161-2.

6. Edmondson R, Broglie JJ, Adcock AF, et al. Three-dimensional cell culture systems and their applications in drug discovery and cell-based biosensors. Assay Drug Dev Technol. 2014;12:207-18.

7. Lin W-W, Karin M. A cytokine-mediated link between innate immunity, inflammation, and cancer. J Clin Invest. 2007;117(5):1175-83.

8. Diakos Cl, Charles KA, McMillan DC, Clarke SJ. Cancer-related inflammation and treatment effectiveness. Lancet Oncol. 2014;15(11):e493-503.

9. Brennecke P, Allavena P, Laface I, Mantovani A, Bottazzi B. Inflammatory and innate immune cells in cancer microenvironment and progression. Cancer Immunology: Springer; 2015. p. 9-28.

10. Mocellin S, Rossi CR, Pilati P, Nitti D. Tumor necrosis factor, cancer and anticancer therapy. Cytokine Growth Factor Rev. 2005;16(1):35-53.

11. Sethi G, Sung B, Aggarwal BB. TNF: a master switch for inflammation to cancer. Front Biosci. 2008;13(2):5094-107.

12. Bertazza L, Mocellin S. The dual role of tumor necrosis factor (TNF) in cancer biology. Curr Med Chem. 2010;17(29):3337-52.

13. Li C-W, Xia W, Huo L, Lim S-O, Wu Y, Hsu JL, et al. Epithelial-mesenchymal transition induced by TNF-a requires NF-KB-mediated transcriptional upregulation of Twist1. Cancer Res. 2012;72(5):1290-300.

14. Roberts NJ, Zhou S, Diaz LA Jr, Holdhoff M. Systemic use of tumor necrosis factor alpha as an anticancer agent. Oncotarget. 2011;2(10):739.

15. Wang $X$, Lin $Y$. Tumor necrosis factor and cancer, buddies or foes? 1. Acta Pharmacol Sin. 2008;29(11):1275-88.

16. Katanov C, Lerrer S, Liubomirski Y, Leider-Trejo L, Meshel T, Bar J, et al. Regulation of the inflammatory profile of stromal cells in human breast cancer: prominent roles for TNF-a and the NF-kB pathway. Stem Cell Res Ther. 2015;6(1):87.

17. Zidi I, Mestiri S, Bartegi A, Amor NB. TNF- $a$ and its inhibitors in cancer. Med Oncol. 2010;27(2):185-98.

18. Madhusudan S, Foster M, Muthuramalingam SR, Braybrooke JP, Wilner S,

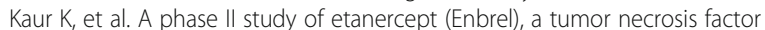
a inhibitor in patients with metastatic breast cancer. Clin Cancer Res. 2004; 10(19):6528-34.

19. Lu J, Zeng H, Liang Z, Chen L, Zhang L, Zhang H, et al. Network modelling reveals the mechanism underlying colitis-associated colon cancer and identifies novel combinatorial anti-cancer targets. Sci Rep. 2015;5:14739.

20. Moshayedi M, Barneh F, Haghjooy-Javanmard S, Sadeghi HM, Eskandari N, Sabzghabaee AM. A rapid and sensitive method for EphB4 identification as a diagnostic and therapeutic biomarker in invasive breast cancer. J Cancer Res Ther. 2016;12(1):188.

21. Schmittgen TD, Livak KJ. Analyzing real-time PCR data by the comparative C T method. Nat Protoc. 2008;3(6):1101

22. Su S, Liu Q, Chen J, Chen J, Chen F, He C, et al. A positive feedback loop between mesenchymal-like cancer cells and macrophages is essential to breast cancer metastasis. Cancer Cell. 2014;25(5):605-20.

23. Kuleshov MV, Jones MR, Rouillard AD, Fernandez NF, Duan Q, Wang Z, et al. Enrichr: a comprehensive gene set enrichment analysis web server 2016 update. Nucleic Acids Res. 2016:44:W90.

24. Dondossola E, Dobroff AS, Marchiò S, Cardó-Vila M, Hosoya H, Libutti SK, et al. Self-targeting of TNF-releasing cancer cells in preclinical models of primary and metastatic tumors. Proc Natl Acad Sci. 2016;113(8):2223-8.

25. Yeung OW, Lo C-M, Ling C-C, Qi X, Geng W, Li C-X, et al. Alternatively activated (M2) macrophages promote tumour growth and invasiveness in hepatocellular carcinoma. J Hepatol. 2015;62(3):607-16.

26. Vandenbon A, Dinh VH, Mikami N, Kitagawa Y, Teraguchi S, Ohkura N, et al. Immuno-navigator, a batch-corrected coexpression database, reveals cell type-specific gene networks in the immune system. Proc Natl Acad Sci. 2016;113(17):E2393-E402.

27. Mercogliano MF, Bruni S, Elizalde PV, Schillaci R. Tumor necrosis factor a blockade: an opportunity to tackle breast Cancer. Front Oncol. 2020;10.

28. Neri D, Sondel PM. Immunocytokines for cancer treatment: past, present and future. Curr Opin Immunol. 2016;40:96-102.

29. Li D, Ji H, Niu X, Yin L, Wang Y, Gu Y, et al. Tumor-associated macrophages secrete CC-chemokine ligand 2 and induce tamoxifen resistance by activating PI3K/Akt/mTOR in breast cancer. Cancer Sci. 2020;111(1):47.
30. Usman MW, Gao J, Zheng T, Rui C, Li T, Bian X, et al. Macrophages confer resistance to PI3K inhibitor GDC-0941 in breast cancer through the activation of NF-kB signaling. Cell Death Dis. 2018;9(8):1-12.

31. Ruffell B, Coussens LM. Macrophages and therapeutic resistance in cancer. Cancer Cell. 2015;27(4):462-72.

32. Yu H, Kortylewski M, Pardoll D. Tumour immunology: crosstalk between cancer and immune cells: role of STAT3 in the tumour microenvironment. Nat Rev Immunol. 2007;7(1):41.

33. Grivennikov SI, Karin M. Dangerous liaisons: STAT3 and NF-KB collaboration and crosstalk in cancer. Cytokine Growth Factor Rev. 2010;21(1):11-9.

34. Banerjee $\mathrm{K}$, Resat $\mathrm{H}$. Constitutive activation of STAT 3 in breast cancer cells: a review. Int J Cancer. 2016;138(11):2570-8.

35. Lee H, Herrmann A, Deng J-H, Kujawski M, Niu G, Li Z, et al. Persistently activated Stat3 maintains constitutive NF-kB activity in tumors. Cancer Cell. 2009;15(4):283-93.

\section{Publisher's Note}

Springer Nature remains neutral with regard to jurisdictional claims in published maps and institutional affiliations.

Ready to submit your research? Choose BMC and benefit from:

- fast, convenient online submission

- thorough peer review by experienced researchers in your field

- rapid publication on acceptance

- support for research data, including large and complex data types

- gold Open Access which fosters wider collaboration and increased citations

- maximum visibility for your research: over $100 \mathrm{M}$ website views per year

At $\mathrm{BMC}$, research is always in progress.

Learn more biomedcentral.com/submissions 